\title{
Acometimento oral no pênfigo vulgar
}

\section{Oral involvement in pemphigus vulgaris}

\author{
Ivan Dieb Miziara', João Aragão Ximenes Filho², \\ Fernando César Ribeiro ${ }^{2}$, Ana Laura Brandão ${ }^{3}$
}

Palavras-chave: pênfigo/epidemiologia, diagnóstico/métodos, avaliação de resultados.

Key words: pemphigus/epidemiology, diagnosis/methods, outcome assessment.

\section{Resumo / Summary}

Pênfigo Vulgar (PV) é uma doença vesicobolhosa caracterizada pela presença de auto-anticorpos contra moléculas de adesão intraepidérmicas. Objetivo: Identificar as características do PV em cada sexo, formas de apresentação, idade de acometimento, métodos de diagnóstico e tratamento utilizado. Forma de Estudo: coorte histórica. Casuística e Método: Pacientes com PV acompanhados no Ambulatório de O torrinolaringologia do HC-FMUSP entre 1990 e 2001. Resultados: Dos 23 pacientes, 91,3\% foram mulheres e $8,7 \%$ homens ( $p<0,01$ ), relação de $9: 1$. A idade ao diagnóstico variou entre 26 e 80 anos, com média de $53 \pm 18,4$ anos. Nos homens, o PV manifestou-se 24,6 \pm 5,9 anos mais precocemente do que nas mulheres $(p=0,026)$. Os principais achados ao exame foram lesões exulceradas na mucosa oral, semelhantes a aftas. Biópsia foi utilizada em $95 \%$ dos casos, imunofluorescência direta em $17,4 \%$ e indireta em $8,7 \%$. A prednisona foi a opção terapêutica em $78 \%$ dos pacientes, enquanto o deflazacort foi usado em $22 \%$. A dapsona foi associada em 4 casos. Obtivemos controle da doença em $74 \%$ dos casos e perda do seguimento após melhora parcial em $13 \%$ dos pacientes. Observamos maior dificuldade no controle da doença no sexo masculino $(p=0,04)$. Conclusões: 0 sexo feminino prevaleceu sobre o masculino. A idade ao diagnóstico foi maior nas mulheres. 0 tratamento de escolha foi a prednisona. O PV foi mais facilmente controlado nas mulheres.

\begin{abstract}
D emphigus vulgaris (PV) is a bullous disease characterized by the presence of autoantibodies against intra-epidermal adhesion molecules. Aim: The aim of this study was to identify the characteristics of PV in each gender, forms of clinical presentation, age, diagnosis methods and management. Study Design: historical cohort. Method: Patients with PV treated in Otolaryngology Department from HC-FMUSP between 1990 and 2001. Results: From 23 patients, 91,3\% were women and $8,7 \%$ men $(p<0,01)$, proportion of $9: 1$. The age of diagnosis varied between 26 and 80 years, with average of $53 \pm 18,4$ years. In males, PV begun $24,6 \pm$ 5,9 years earlier than in females $(p=0,026)$. The most frequently sign in physical examination were bullous lesions in oral mucosa. Biopsy was used in $95 \%$ of the cases, direct immunofluorescence in $17,4 \%$ and indirect $8,7 \%$. Prednisona was the therapeutic option in $78 \%$ of the patients, while deflazacort was used in $22 \%$. Dapsona was associated in 4 cases. We obtained control of the disease in $74 \%$ of the cases and loss of the continuation after partial improvement in more $13 \%$ of the patients. We observed larger difficulty in disease control in males $(p=0,04)$. Conclusions: The females prevailed over males. The age of diagnosis was higher in women. The treatment of choice was prednisona. PV was more easily controlled in females.
\end{abstract}

\footnotetext{
${ }^{1}$ Médico Assistente Doutor da Divisão de Clínica O torrinolaringológica do Hospital das Clínicas da Faculdade de Medicina da USP. ${ }^{2}$ Doutorando do Curso de Pós-Graduação em Otorrinolaringologia da FMUSP.

${ }^{3}$ Médico Colaborador da Divisão de Clínica O torrinolaringologia do Hospital das Clínicas da FMUSP.

Trabalho Realizado no Ambulatório de Otorrinolaringologia da Faculdade de Medicina da Universidade de São Paulo (FMUSP). Endereço para correspondência: João Aragão Ximenes Filho - Rua Assunção, 773 Fortaleza CE 60050-011. Tel (0xx85) 231-6296 - E-mail: joaoximenesf@bol.com.br

Artigo recebido em 20 de dezembro de 2002. Artigo aceito em 07 de fevereiro de 2003.
} 


\section{INTRODUÇÃO}

O Pênfigo Vulgar (PV) é uma doença vesicobolhosa crônica de natureza auto-imune. Acomete pele e mucosas e caracteriza-se pela presença de auto-anticorpos contra proteínas desmossômicas encontrado nas junções epiteliais dos tecidos de revestimento ${ }^{1}$. A faixa etária mais acometida encontra-se entre a $5^{\mathrm{a}}$ e $6^{\mathrm{a}}$ décadas de vida, sendo menos freqüente após os 70 anos e em crianças ${ }^{2,3}$. Existem discordância na literatura quanto ao acometimento preferencialmente em mulheres. Wanke et al. ${ }^{4}$ (1990) afirmam que acomete homens e mulheres igualmente. No entanto, Robinson et al. ${ }^{5}$ (1997) apresentam uma proporção de 9 mulheres para cada grupo de 3 homens.

Em geral, a enfermidade tem início com lesões orais, vindo posteriormente a acometer a pele. 0 diagnóstico é firmado pelo exame histopatológico que mostra bolhas acantolíticas intra-epidérmicas baixas, logo acima da zona da membrana basal. A imunofluorescência direta auxilia no diagnóstico ao revelar a presença de imunoglobulinas $G$ (IgG) nos espaços intercelulares da epiderme. 0 diagnóstico diferencial com o pênfigo foliáceo é feito clinicamente, pois esse último não acomete mucosas ${ }^{6}$. O PV apresentava, antes do surgimento dos corticosteróides, mortalidade em torno de $90 \%$. Com os avanços nas opções terapêuticas, este índice encontra-se atualmente abaixo de 20\%4,5. Dentre os corticosteróides, a prednisona e o deflazacort têm sido utilizados como tratamento de eleição desta afecção?. O utras opções são a dapsona ${ }^{3}$, a aureoterapia oral ${ }^{8,9}$, ciclofosfamida ${ }^{10,11}$, entre outras menos utilizadas.

0 objetivo deste trabalho foi comparar as características do PV em cada sexo, como prevalência, idade mais freqüente de acometimento, formas clínicas de apresentação, tempo de sintomas antes do diagnóstico, além dos métodos usados no diagnóstico. Atenção especial foi dada as opções de tratamento adotadas e os resultados obtidos.

\section{CASUÍSTICA E MÉTODO}

\section{Casuística}

Foram revisados os prontuários dos pacientes com diagnóstico de Pênfigo Vulgar acompanhados e tratados no Ambulatório de O torrinolaringologia do Hospital das Clínicas da Faculdade de Medicina da Universidade de São Paulo no período entre janeiro de 1990 e dezembro de 2001.

Foram incluídos apenas os prontuários completa e corretamente preenchidos e com seguimento no ambulatório por 30 dias ou mais. Prontuários com dados incompletos, sem diagnóstico final firmado ou com seguimento menor que 30 dias foram excluídos.

\section{Método}

Utilizamos uma ficha de coleta de dados onde se apresentavam as variáveis em estudo. Foram registrados os dados referentes à identificação do doente, idade ao diagnóstico, sexo, raça, tempo de sintomas antes do diagnóstico, exames complementares utilizados, tratamento adotado, seguimento e resultados obtidos.

Dividimos nossa população em grupos conforme 0 sexo para compararmos as características do PV em homens e mulheres e observar se existem diferenças nos parâmetros estudados. As comparações entre os dados contínuos (idade, tempo) foram feitas pelo teste $t$ de Student. Nas demais comparações entre os grupos masculino e feminino, utilizamos o teste do qui-quadrado ou o teste exato de Fischer. Os dados foram analisados nos programas Microsoft Excel 2000 e SPSS 10.0 for Windows. Foi considerado índice de significância de $95 \%(p<0,05)$.

\section{RESULTADOS}

No presente estudo, foram identificadas 23 pacientes com diagnóstico de Pênfigo Vulgar, sendo 21 (91,3\%) do sexo feminino e $2(8,7 \%)$ do sexo masculino. Quando comparamos com os dados de Wanke et al. ${ }^{4}$, onde não há predominância por sexo, encontramos diferença estatisticamente significante $(p<0,01)$. Quando comparamos com a relação de 9 mulheres para três homens ${ }^{5}$, observamos que nossa amostra não diferiu estatisticamente $(p=0,12)$ (GráfiCO 1).

A idade ao diagnóstico variou entre 26 e 80 anos, com média de $53 \pm 18,4$ anos. No sexo masculino, a idade média foi $30,5 \pm 6,36$, enquanto nas mulheres foi $55,1 \pm$ 17,7 anos. Comparando a idade entre os sexos, observamos que, no sexo masculino, o PV se manifestou $24,6 \pm 5,9$ anos mais precocemente do que no sexo feminino $(p=0,026)$ (Gráfico 2).

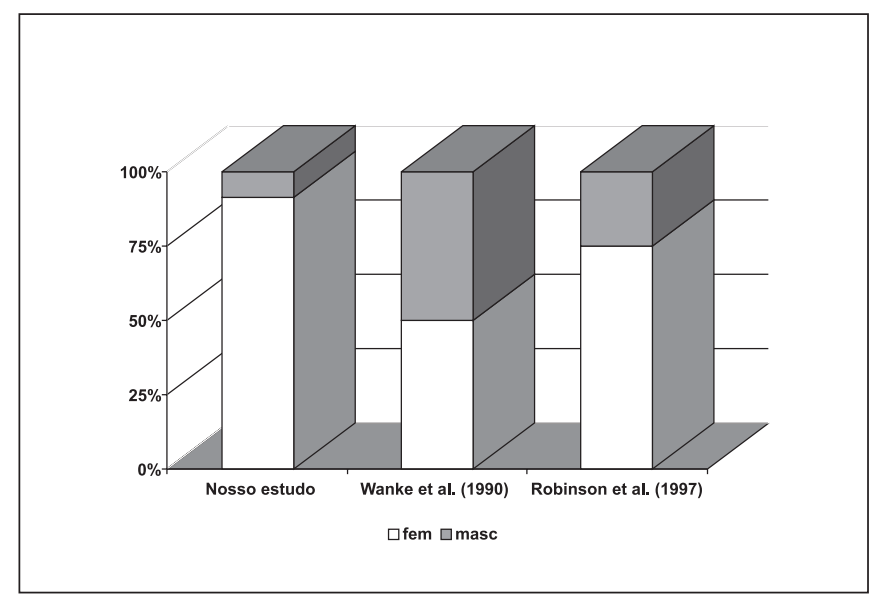

Gráfico 1. Comparação da prevalência de Pênfigo Vulgar entre os sexos no nosso estudo e na literatura. 


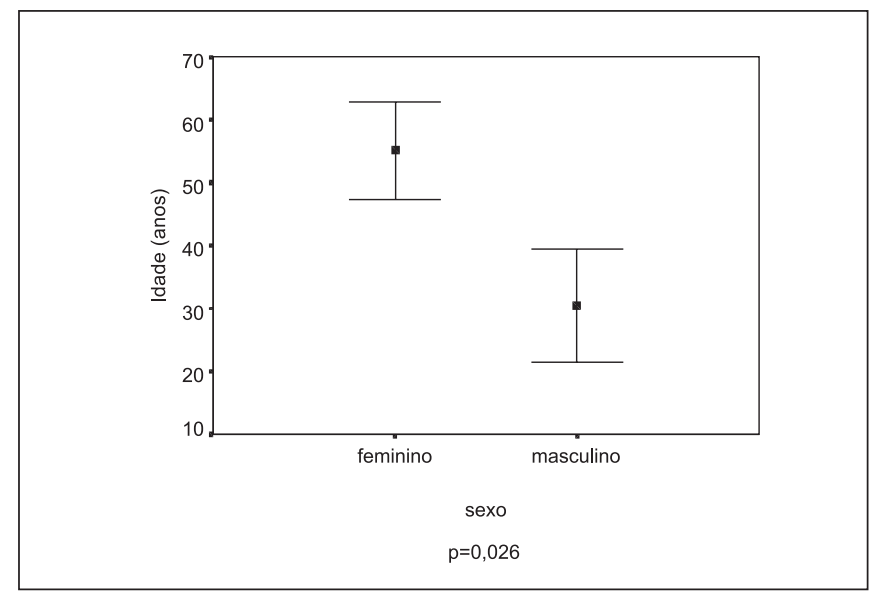

Gráfico 2. Comparação entre as médias das idades ao diagnóstico de Pênfigo Vulgar nos sexos, mostrando ocorreu mais precocemente que no sexo masculino.

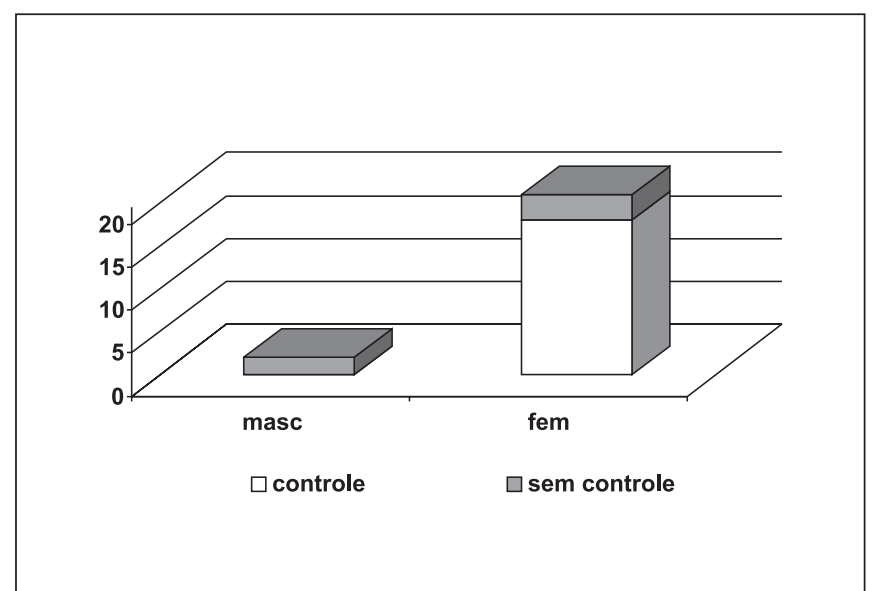

Gráfico 4. Comparação entre os resultados obtidos no tratamento do Pênfigo Vulgar nos sexos, mostrando que no sexo masculino a doença foi de mais difícil controle.

O principal achado ao exame físico foi a presença de lesões exulceradas na mucosa oral, com aspecto semelhante a aftas, sem diferença entre os sexos $(p=0,39)$. Essas lesões podem persistir por semanas a meses. 0 tempo de sintomas antes do diagnóstico variou de 1 a 72 meses, com média de 24,3 $\pm 12,5$ meses (Gráfico 3). Não encontramos diferenças entre os sexos $(p=0,25)$. Os principais exames complementares realizados com finalidade diagnóstica foram: biópsia incisional da lesão oral em 22 casos (95\%) realizada com a utilização de "punch", imunofluorescência direta em 4 casos $(17,4 \%)$ e imunofluo rescência indireta em 2 casos $(8,7 \%)$. Não encontramos diferenças entre os sexos $(p=0,47)$.

A principal modalidade de tratamento utilizada foi a corticoterapia oral, usada em todos os casos. A prednisona foi a opção em 18 pacientes (78\%), enquanto o deflazacort foi usado em 5 (22\%). Um dos pacientes usou inicialmente

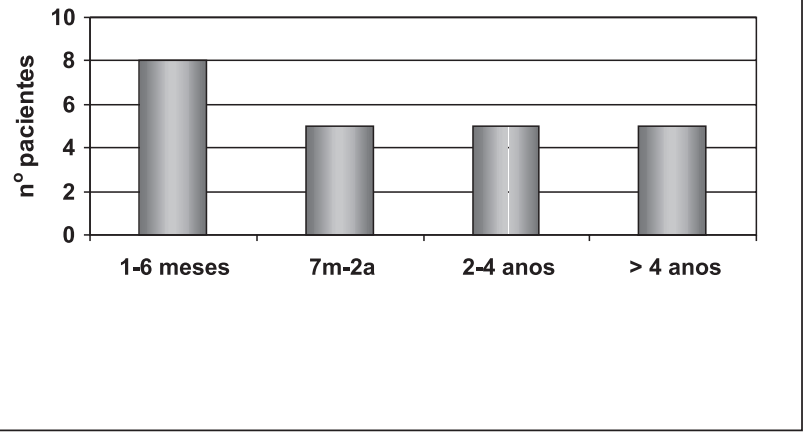

Gráfico 3. Tempo de doença antes do diagnóstico de Pênfigo Vulgar.

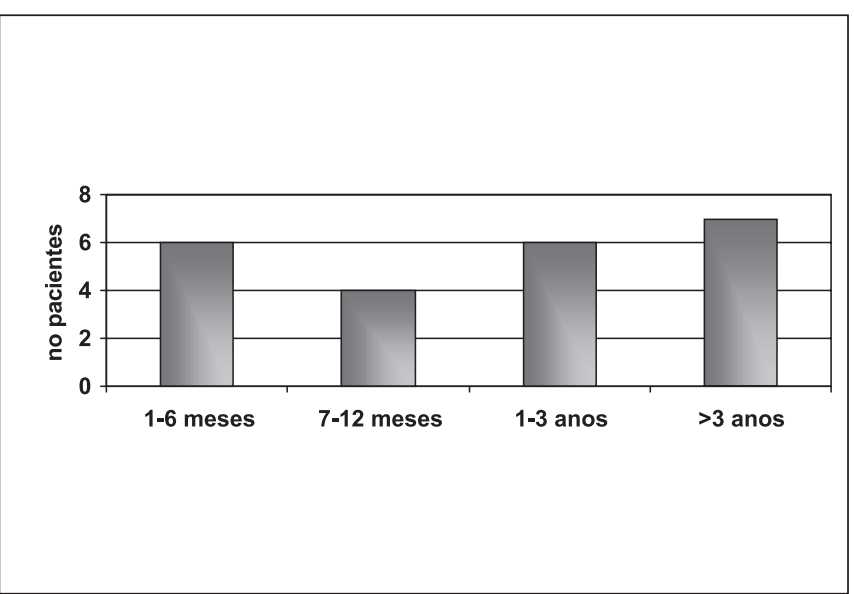

Gráfico 5. Tempo de seguimento dos doentes em nosso ambulatório.

prednisona, mas tivemos que substituir por deflazacort em decorrências dos efeitos colaterais. Outra medicação utilizada foi a dapsona, empregada em 4 pacientes $(17,4 \%)$. Não encontramos diferenças entre os sexos quanto às opções de tratamento utilizadas $(p=0,09)$.

Os resultados aos tratamentos instituídos foram analisados separadamente entre homens e mulheres (Gráfico 4), mostrando haver maior dificuldade no controle da doença no sexo masculino $(p=0,04)$. 0 tempo de seguimento dos pacientes variou de 6 a 84 meses, com média de $28,5 \pm$ 14,9 (Gráfico 5), sem diferenças entre os sexos $(p=0,13)$.

\section{DISCUSSÃO}

O Pênfigo vulgar é uma doença considerada pela maioria dos autores de prognóstico sombrio, podendo ser mortal acima de $90 \%$ dos casos não tratados ${ }^{12}$. 0 diagnóstico 
e tratamento são de origem multidisciplinar, pois fazem parte dos mesmos Dentistas, O torrinolaringologistas e Dermatologistas. Neste estudo, buscamos avaliar as características epidemiológicas dos indivíduos diagnosticados e tratados por PV oral em nosso serviço, comparando os sexos e observar os métodos diagnósticos, opções terapêuticas utilizadas e a resposta ao tratamento destes indivíduos.

Quanto ao número de pacientes estudados, observamos que outros autores apresentam casuística semelhante. Jimenez-Barea e Magnin ${ }^{8}$ (1990) apresentam 19 pacientes com PV, Maceira e Marques ${ }^{13}$ (1990) detectam em nove anos 17 casos de PV, enquanto Fernandez e Perez ${ }^{14}$ (2001) estudam 41 indivíduos com PV em 20 anos num grande hospital no Rio de Janeiro. Nesta revisão, detectamos em 12 anos de estudo 23 doentes portadores de PV, todos com acometimento oral, o que demonstra casuística compatível com a da literatura.

Os principais resultados que obtivemos foram as diferenças entre homens e mulheres na prevalência, idade de apresentação e na resposta ao tratamento. Existe uma discrepância na literatura sobre a prevalência de PV nos sexos. Nossa casuística corrobora aqueles autores que afirmam ser o sexo feminino mais acometido e apresentamos uma relação de 9:1, semelhante a outras doenças auto-imunes. Q uanto à idade e a resposta ao tratamento, não encontramos estudos que demonstrassem diferença entre os sexos. Aqui, observamos que, nos homens, o PV manifestou-se mais precocemente e teve um controle mais difícil quando comparado ao sexo feminino. No entanto, como tivemos apenas dois indivíduos do sexo masculino, fica difícil a extrapolação desses resultados. Seriam, então, necessários estudos multicêntricos, com a inclusão de um maior número de pacientes, para a confirmação destas diferenças de manifestação do PV entre homens e mulheres.

O diagnóstico de PV na cavidade oral é realizado através de exame anatomopatológico onde são evidenciadas células acantolíticas arredondadas e exfoliativas ${ }^{15}$. A imunofluorescência direta (ID), onde ocorre a detecção dos anticorpos no material biopsiado, tem sido utilizada para aumentar a sensibilidade da biópsia. Marceira e Marques $^{13}$ (1990) demonstram que este método tem alta sensibilidade no diagnóstico de PV. Schneider e Schneider ${ }^{15}$ (1998), porém, afirmam que a ID tem pouca utilidade prática. Em nosso estudo, utilizamos a ID de rotina conjuntamente com o estudo anatomopatológico com o objetivo de aumentar a sensibilidade deste último método.

A imunofluorescência indireta, onde a detecção dos anticorpos ocorre no sangue periférico, tem também sido utilizada em portadores de PV. Parece ter positividade de apenas $66,6 \%$ no diagnóstico desta doença ${ }^{13}$, porém é muito útil no monitoramento da doença durante tratamento clínico com corticosteróides ${ }^{15}$. Em nosso serviço, utilizamos este método como preditivo de atividade da doença, orientandonos na retirada da medicação antipênfigo.
Diversas opções têm sido utilizadas no tratamento do PV. A prednisona é, contudo, a droga de escolha pela maioria dos autores $3,4,16$, apresentando bons resultados no controle da doença. Usualmente, esta droga é utilizada por períodos prolongados, durante meses ou anos, até a negativação da imunofluorescência indireta. Assim, eventualmente, surgem efeitos colaterais importantes, necessitando de outras opções terapêuticas.

Em nosso estudo, observamos bons resultados com a prednisona. Porém, em algumas situações, tivemos a necessidade em aumentarmos sua dose para níveis superiores a $1 \mathrm{mg} / \mathrm{kg} /$ dia para o controle da doença. Nestes casos, optamos pela associação da dapsona, na dose de $100 \mathrm{mg} / \mathrm{dia}^{3}$, com bons resultados. Outros autores têm utilizado sais de ouro ${ }^{8,9}$, também com bons resultados. No entanto, o custo elevado e os efeitos colaterais impediram a popularização do seu uso. Ciclofosfamida também tem sido proposta nestes casos, podendo ser utilizada em pulsos endovenosos mensais ou por via oral ${ }^{11}$. No entanto, a primeira necessita de internação em hospital dia e apresenta efeitos colaterais importantes, somente justificada sua utilização em casos graves. A forma oral apresenta importante efeito imunossupressor, exigindo maior cuidado em sua prescrição.

Alguns pacientes apresentam, no entanto, efeitos adversos mesmo em doses baixas de prednisona (menores que $1 \mathrm{mg} / \mathrm{kg} / \mathrm{dia}$ ). Nestes casos, o deflazacort em doses não superiores a $60 \mathrm{mg} / \mathrm{dia}$. Esta droga, apesar de ter maior custo, apresenta-se como a melhor opção nestas situações.

Por fim, em nosso estudo, mantivemos seguimento por até 84 meses, com média de mais de dois anos. Isto é necessário uma vez que a terapia controla, mas não cura a doença ${ }^{12}$, sendo importante 0 acompanhamento destes pacientes por períodos prolongados, bem como o esclarecimento da história natural da doença aos indivíduos acometidos.

\section{CONCLUSÕES}

As principais conclusões deste estudo foram:

1. O sexo feminino prevaleceu sobre o masculino, com relação de 9:1;

2. A idade ao diagnóstico foi maior nas mulheres;

3. A lesão exulcerada foi a manifestação oral mais freqüente;

4. 0 tratamento de escolha foi a prednisona por seu alto percentual de controle e seu baixo custo;

5. O PV foi mais facilmente controlado no sexo feminino.

\section{REFERÊNCIAS BIBLIOGRÁFICAS}

1. Dabelsteen E. Molecular biological aspects of acquired bullous diseases. Crit Rev Oral Biol Med 1998; 9(2):162-178.

2. Gorsky M, Raviv M, Raviv E. Pemphigus vulgaris in adolescence. A case presentation and review of the literature. Oral Surg Oral Med Oral Pathol 1994; 77(6):620-622. 
3. Miziara ID, Costa EG, Miniti A. O uso da dapsona associado à prednisona no tratamento do pênfigo vulgar. Rev Bras Otorrinolaringol 1994; 60(4):280-286.

4. Wanke CF, Silva MM, Brandão MG, Maceira J. Tratamento de pênfigos: revisão de 31 casos. An Bras Dermastol 1990; 65(3): 119-122.

5. Robinson JC, Lozada-Nur F, Frieden I. Oral pemphigus vulgaris: a review of the literature and a report on the management of 12 cases. Oral Surg Oral Med Oral Pathol Oral Radiol Endod 1997; 84(4): 349-355.

6. Sampaio SAP, Rivitti EA. Erupções vésico-bolhoras. In: Dermatologia. Ed. São Paulo: Artes Médicas; 1998. pp. 229-240.

7. Mignogna MD, Lo Muzio L, Mignogna RE, Carbone R, Ruoppo E, Bucci E. Oral pemphigus: long term behaviour and clinical response to treatment with deflazacort in sixteen cases. J Oral Pathol Med 2000; 29(4):145-152.

8. Jimenez-Barea LF, Magnin PH. Control evolutivo del penfigo con aureoterapia oral. Rev Arg Derm 1990; 71: 33-38.

9. Magnin PH, Spillman DH, Ixilia MR. Aureoterapia oral en el penfigo. Rev Arg Derm 1988; 69: 184-188.
10. Engineer L, Norton LA, Ahmed AR. Nail involvement in pemphigus vulgaris. J Am Acad Dermatol 2000; 43(3):529-535.

11. Fox LP, Pandya AG. Pulse intravenous cyclophosphamide therapy for dermatologic disorders. Dermatol Clin 2000; 18(3):459-473.

12. Talhari EA, Marcucci G. Pênfigo vulgar: aspectos estomatológicos. Rev Assoc Paul Cirurg Dent 1986; 40(4): 290-295.

13. Maceira JP, Marques AS. Estudo de imunofluorescência em 40 casos observados no Hospital Universitário Federal do Rio de Janeiro. An Bras Dermatol 1990; 65(3):113-115.

14. Fernandez NC, Perez M. Treatment of pemphigus vulgaris and pemphigus foliaceus: experience with 71 patients over a 20 years pediod. Rev Inst Med Trop S Paulo 2001; 43(1):33-36.

15. Schneider LC, Schneider AE. Diagnosis of oral ulcers. Mount Sinai J Med 1998; 65(5-6):383-387.

16. Fantasia JE. Diagnosis and treatment of common oral lesions found in the elderly. Clinical Decision-Making in Geriatric Dentistry 1997; 41(4):877-890. 